\title{
Some lemmata on the perturbation of the spectrum
}

\author{
Alexander I. Nazarov*
}

\begin{abstract}
We give some sufficient conditions for preserving of the second term in the spectral asymptotics of a compact operator under the perturbation of the metrics in the Hilbert space.
\end{abstract}

It is well known, see, e.g., [1, Lemma 1.16], that the one-term powertype spectral asymptotics of a compact operator in the Hilbert space does not change under compact perturbation of the metrics of the space. The problem of preserving of the two-term asymptotics is much more sensitive and complicated. Here we give some sufficient conditions for this. These results can be applied in the spectral analysis of some integro-differential operators arising in the theory of Gaussian random processes, see [3].

In what follows we denote by $c$ any absolute constant.

Lemma 1 Let $\mathcal{K}$ and $\mathcal{B}$ be self-adjoint compact operators in the Hilbert space $\mathcal{H}$. Suppose that $\mathcal{K}$ and $\mathcal{I}+\mathcal{B}$ are positive. Denote by $\lambda_{n}$ the eigenvalues of $\mathcal{K}$ enumerated in the decreasing order taking into account the multiplicities, and by $h_{n}$ corresponding normalized eigenfunctions. Finally, suppose that

$$
\lambda_{n}=\left(a n+b+O\left(n^{-\delta}\right)\right)^{-B}, \quad\left\|\mathcal{B} h_{n}\right\|_{\mathcal{H}} \leq c n^{-(1+\delta)},
$$

as $n \rightarrow \infty$, where $a, B, \delta>0, b \in \mathbb{R}$. Then the eigenvalues $\boldsymbol{\lambda}_{n}$ of generalized eigenproblem

$$
\mathcal{K} \mathbf{h}_{n}=\boldsymbol{\lambda}_{n}\left(\mathbf{h}_{n}+\mathcal{B} \mathbf{h}_{n}\right)
$$

*St.Petersburg Department of Steklov Institute, Fontanka 27, St.Petersburg, 191023, Russia, and St.Petersburg State University, Universitetskii pr. 28, St.Petersburg, 198504, Russia. E-mail: al.il.nazarov@gmail.com. 
have the same two-term asymptotics as $n \rightarrow \infty$ :

$$
\boldsymbol{\lambda}_{n}=\left(a n+b+O\left(n^{-\delta}\right)\right)^{-B} .
$$

Proof. We introduce new scalar products in $\mathcal{H}$ :

$$
\langle h, g\rangle:=(h+\mathcal{B} h, g)_{\mathcal{H}} .
$$

It is easy to see that corresponding norm $\|h\|:=\langle h, h\rangle^{\frac{1}{2}}$ is equivalent to original one. Denote by $\mathbb{H}$ the space $\mathcal{H}$ with new scalar product. Then the sesquilinear form $(\mathcal{K} h, g)_{\mathcal{H}}$ generates a compact positive self-adjoint operator $\mathbb{B}$ such that

$$
\langle\mathbb{B} h, g\rangle=(\mathcal{K} h, g)_{\mathcal{H}}, \quad h, g \in \mathbb{H},
$$

and the generalized eigenproblem (2) is reduced to the standard eigenproblem for the operator $\mathbb{B}$ in $\mathbb{H}$.

Recall some elementary facts from the theory of spectral measure, see Ch. 5 in [2]. The spectral measure $d \mathcal{E}(t)$ associated with $\mathbb{B}$ generates the family of scalar measures

$$
d e_{h}(t):=\langle d \mathcal{E}(t) h, h\rangle, \quad h \in \mathbb{H} .
$$

Moreover, the following obvious formulae hold for arbitrary $h \in \mathbb{H}$ :

$$
\|h\|^{2}=\int_{\mathbb{R}} d e_{h}(t), \quad\|\mathbb{B} h-\lambda h\|^{2}=\int_{\mathbb{R}}(t-\lambda)^{2} d e_{h}(t) .
$$

If we assume that an interval $\Delta=(\lambda-\delta, \lambda+\delta)$ is free of the spectrum of $\mathbb{B}$ then we have for any $h \in \mathbb{H}$

$$
\|\mathbb{B} h-\lambda h\|^{2}=\int_{\mathbb{R} \backslash \Delta}(t-\lambda)^{2} d e_{h}(t) \geq \delta^{2} \int_{\mathbb{R} \backslash \Delta} d e_{h}(t)=\delta^{2}\|h\|^{2} .
$$

Now we set $\lambda=\lambda_{n}, h=h_{n}$. For any $g \in \mathbb{H}$ we have

$$
\begin{aligned}
\left|\left\langle\mathbb{B} h_{n}-\lambda_{n} h_{n}, g\right\rangle\right| & =\left|\left(\mathcal{K} h_{n}, g\right)_{\mathcal{H}}-\lambda_{n}\left(h_{n}+\mathcal{B} h_{n}, g\right)_{\mathcal{H}}\right| \\
& =\lambda_{n}\left|\left(\mathcal{B} h_{n}, g\right)_{\mathcal{H}}\right| \leq \lambda_{n}\left\|\mathcal{B} h_{n}\right\|_{\mathcal{H}}\|g\|_{\mathcal{H}} \leq \frac{c_{1}}{n^{1+\delta}} \lambda_{n}\|g\|,
\end{aligned}
$$

and therefore

$$
\left\|\mathbb{B} h_{n}-\lambda_{n} h_{n}\right\| \leq \frac{c_{1}}{n^{1+\delta}} \lambda_{n} \leq \frac{c_{2}}{n^{1+\delta}} \lambda_{n}\left\|h_{n}\right\| .
$$


Comparing this inequality with (44) we see that the interval

$$
\Delta_{n}=\left(\lambda_{n}\left(1-c_{2} n^{-(1+\delta)}\right), \lambda_{n}\left(1+c_{2} n^{-(1+\delta)}\right)\right)
$$

contains an eigenvalue $\boldsymbol{\lambda}$ of the generalized eigenproblem (2).

By (1), intervals $\Delta_{n}$ and $\Delta_{n+1}$ do not intersect for $n$ sufficiently large. Repeating previous argument for $\varepsilon \mathcal{B}$ instead of $\mathcal{B}, \varepsilon \in[0,1]$, we notice that the eigenvalues depend continuously on $\varepsilon$ and conclude that for large $n$ the interval $\Delta_{n}$ contains just $\boldsymbol{\lambda}_{n}$. This yields (3).

This result is quite simple but the assumption (11) is very restrictive. The following theorem gives a "more pointwise" condition which is, however, globally weaker.

Theorem 1 In Lemma 1, suppose that instead of (1) the following relations hold:

$$
\lambda_{n}=\left(a n+b+O\left(n^{-\delta}\right)\right)^{-B}, \quad\left|\left(\mathcal{B} h_{n}, h_{m}\right)_{\mathcal{H}}\right| \leq c(m n)^{-\frac{1+\delta}{2}} .
$$

Then (3) also holds.

Proof. First, we notice that we can write $\mathcal{B}=\mathcal{B}_{+}+\mathcal{B}_{-}$, where $\mathcal{B}_{+} \geq 0$ and $\mathcal{B}_{-} \leq 0$. By the min-max principle (see, e.g., [1, Appendix 1], we have $\boldsymbol{\lambda}_{n}^{+} \leq \boldsymbol{\lambda}_{n} \leq \boldsymbol{\lambda}_{n}^{-}$, where $\boldsymbol{\lambda}_{n}^{ \pm}$are eigenvalues of the problems

$$
\mathcal{K} \mathbf{h}_{n}^{+}=\boldsymbol{\lambda}_{n}^{+}\left(\mathbf{h}_{n}^{+}+\mathcal{B}_{+} \mathbf{h}_{n}^{+}\right) ; \quad \mathcal{K} \mathbf{h}_{n}^{-}=\boldsymbol{\lambda}_{n}^{-}\left(\mathbf{h}_{n}^{-}+\mathcal{B}_{-} \mathbf{h}_{n}^{-}\right) .
$$

So, it suffices to consider two cases: positive $\mathcal{B}$ and negative $\mathcal{B}$.

1. Let $\mathcal{B}$ be positive. Then evidently $\boldsymbol{\lambda}_{n} \leq \lambda_{n}$. On the other hand, the min-max principle gives $\boldsymbol{\lambda}_{n} \geq \widehat{\lambda}_{n}$, where $\widehat{\lambda}_{k}$ are the eigenvalues of generalized (finite-dimensional) eigenproblem

$$
\widehat{P}_{n} \mathcal{K} \widehat{P}_{n} \widehat{h}_{k}=\widehat{\lambda}_{k}\left(\widehat{h}_{k}+\widehat{P}_{n} \mathcal{B} \widehat{P}_{n} \widehat{h}_{k}\right)
$$

and $\widehat{P}_{n}$ is the orthoprojector onto the span of $\widehat{\mathcal{H}}_{n}=\operatorname{Span}\left\{h_{k}\right\}, k \leq n$.

Let $\widehat{x} \in \widehat{\mathcal{H}}_{n}$ be the minimizer of the Rayleigh quotient

$$
J(x)=\frac{(\mathcal{K} x, x)_{\mathcal{H}}}{(x, x)_{\mathcal{H}}+(\mathcal{B} x, x)_{\mathcal{H}}}
$$


over $\widehat{\mathcal{H}}_{n}$. We derive for $k<n$

$$
0=\frac{1}{2} J^{\prime}\left(\widehat{x} ; h_{k}\right)=\frac{\left.\left(\mathcal{K} \widehat{x}, h_{k}\right)_{\mathcal{H}}-J(\widehat{x}) \cdot\left(\left(\widehat{x}, h_{k}\right)_{\mathcal{H}}+\left(\mathcal{B} \widehat{x}, h_{k}\right)_{\mathcal{H}}\right)\right)}{(\widehat{x}, \widehat{x})_{\mathcal{H}}+(\mathcal{B} \widehat{x}, \widehat{x})_{\mathcal{H}}} .
$$

Therefore,

$$
0=\left(\lambda_{k}-J(\widehat{x})\right) \cdot\left(\widehat{x}, h_{k}\right)_{\mathcal{H}}-J(\widehat{x})\left(\mathcal{B} \widehat{x}, h_{k}\right)_{\mathcal{H}},
$$

i.e.

$$
\left(\widehat{x}, h_{k}\right)_{\mathcal{H}}=\frac{J(\widehat{x})}{\lambda_{k}-J(\widehat{x})}\left(\mathcal{B} \widehat{x}, h_{k}\right)_{\mathcal{H}} .
$$

Since $J\left(h_{n}\right) \leq \lambda_{n}$, we have $J(\widehat{x}) \leq \lambda_{n}$. So, for any $k<n$

$$
\widehat{a}_{k}:=\left|\left(\widehat{x}, h_{k}\right)_{\mathcal{H}}\right| \leq \frac{\lambda_{n}}{\lambda_{k}-\lambda_{n}} \sum_{m=1}^{n} \widehat{a}_{m} \cdot \frac{c}{(k m)^{\frac{1+\delta}{2}}} .
$$

This implies

$$
\widehat{A}:=\sum_{k=1}^{n} \frac{\widehat{a}_{k}}{k^{\frac{1+\delta}{2}}} \leq \widehat{A} \sum_{k=1}^{n-1} \frac{\lambda_{n}}{\lambda_{k}-\lambda_{n}} \cdot \frac{c}{k^{1+\delta}}+\frac{\widehat{a}_{n}}{n^{\frac{1+\delta}{2}}}=: \widehat{A} \widehat{\mathfrak{C}}+\frac{\widehat{a}_{n}}{n^{\frac{1+\delta}{2}}} .
$$

Notice that for $k<n$

$$
\frac{\lambda_{n}}{\lambda_{k}-\lambda_{n}} \leq c \frac{(k / n)^{B}}{1-(k / n)^{B}}+\frac{c}{n^{\min \{1, B\}}} .
$$

So,

$$
\begin{aligned}
\widehat{\mathfrak{C}} & \leq \frac{c}{n^{1+\delta}} \sum_{k=1}^{n-1} \frac{(k / n)^{B-1-\delta}}{1-(k / n)^{B}}+\frac{c}{n^{\min \{1, B\}}} \\
& \leq \frac{c}{n^{\delta}} \int_{\frac{1}{n}}^{1-\frac{1}{n}} \frac{t^{B-1-\delta}}{1-t^{B}} d t+\frac{c}{n^{\min \{1, B\}}} \leq \frac{c \log (n)}{n^{\min \{1, \delta, B\}}},
\end{aligned}
$$

and for $n$ sufficiently large (77) gives $\widehat{A} \leq c \widehat{a}_{n} n^{-\frac{1+\delta}{2}}$.

Now we calculate

$$
J(\widehat{x}) \geq \frac{\sum_{k=1}^{n} \lambda_{k} \widehat{a}_{k}^{2}}{\sum_{k=1}^{n} \widehat{a}_{k}^{2}+c \sum_{k, m=1}^{n} \frac{\widehat{a}_{k} \widehat{a}_{m}}{(k m)^{\frac{1+\delta}{2}}}} \geq \lambda_{n}\left(1-c n^{-(1+\delta)}\right),
$$


and the statement follows.

2. Let $\mathcal{B}$ be negative. Then evidently $\boldsymbol{\lambda}_{n} \geq \lambda_{n}$. On the other hand, the min-max principle gives $\boldsymbol{\lambda}_{n+k-1} \leq \widetilde{\lambda}_{k}$, where $\widetilde{\lambda}_{k}$ are the eigenvalues of generalized eigenproblem

$$
\widetilde{P}_{n} \mathcal{K} \widetilde{P}_{n} \widetilde{h}_{k}=\widetilde{\lambda}_{k}\left(\widetilde{h}_{k}+\widetilde{P}_{n} \mathcal{B} \widetilde{P}_{n} \widetilde{h}_{k}\right),
$$

and $\widetilde{P}_{n}$ is the orthoprojector onto $\widetilde{\mathcal{H}}_{n}=\operatorname{Span}\left\{h_{n+k-1}\right\}, k \geq 1$.

Let $\widetilde{x} \in \widetilde{\mathcal{H}}_{n}$ be the maximizer of the Rayleigh quotient (15) over $\widetilde{\mathcal{H}}_{n}$. Just as in the first part, we obtain (6) for $k>n$.

Since $J\left(h_{n}\right) \geq \lambda_{n}$, we have $J(\widetilde{x}) \geq \lambda_{n}$. So, for any $k>n$

$$
\widetilde{a}_{k}:=\left|\left(\widetilde{x}, h_{k}\right)_{\mathcal{H}}\right| \leq \frac{\lambda_{n}}{\lambda_{n}-\lambda_{k}} \sum_{m=n}^{\infty} \widetilde{a}_{m} \cdot \frac{c}{(k m)^{\frac{1+\delta}{2}}} .
$$

This implies

$$
\widetilde{A}:=\sum_{k=n}^{\infty} \frac{\widetilde{a}_{k}}{k^{\frac{1+\delta}{2}}} \leq \widetilde{A} \sum_{k=n+1}^{\infty} \frac{\lambda_{n}}{\lambda_{n}-\lambda_{k}} \cdot \frac{c}{k^{1+\delta}}+\frac{1}{n^{\frac{1+\delta}{2}}}=: \widetilde{A} \widetilde{\mathfrak{C}}+\frac{1}{n^{\frac{1+\delta}{2}}} .
$$

Notice that for $k>n$

$$
\frac{\lambda_{n}}{\lambda_{n}-\lambda_{k}} \leq c \frac{(k / n)^{B}}{(k / n)^{B}-1}+\frac{c}{n^{\min \{1, B\}}} .
$$

So,

$$
\begin{aligned}
\widetilde{\mathfrak{C}^{\mathfrak{*}}} & \leq \frac{c}{n^{1+\delta}} \sum_{k=n+1}^{\infty} \frac{(k / n)^{B-1-\delta}}{(k / n)^{B}-1}+\frac{c}{n^{\min \{1, B\}}} \\
& \leq \frac{c}{n^{\delta}} \int_{1+\frac{1}{n}}^{\infty} \frac{t^{B-1-\delta}}{t^{B}-1} d t+\frac{c}{n^{\min \{1, B\}}} \leq \frac{c \log (n)}{n^{\min \{1, \delta, B\}}},
\end{aligned}
$$

and for $n$ sufficiently large (8) gives $\widetilde{A} \leq c n^{-\frac{1+\delta}{2}}$.

Now we calculate

$$
J(\widetilde{x}) \leq \frac{\sum_{k=n}^{\infty} \lambda_{k} \widetilde{a}_{k}^{2}}{\sum_{k=n}^{\infty} \widetilde{a}_{k}^{2}-c \sum_{k, m=n}^{\infty} \frac{\widetilde{a}_{k} \widetilde{a}_{m}}{(k m)^{\frac{1+\delta}{2}}}} \leq \lambda_{n}\left(1+c n^{-(1+\delta)}\right),
$$

and the statement again follows. 
Remark 2 The results of Lemma 1 and Theorem 1 hold true also in the case where the eigenvalues of the operator $\mathcal{K}$ are organized in two sequences

$$
\lambda_{n}^{(1)}=\left((2 n-1) a+b_{1}+O\left(n^{-\delta}\right)\right)^{-B}, \quad \lambda_{n}^{(2)}=\left(2 n a+b_{2}+O\left(n^{-\delta}\right)\right)^{-B},
$$

as $n \rightarrow \infty$. Such asymptotics is preserved under the same assumptions on the perturbation operator $\mathcal{B}$.

\section{References}

[1] Birman, M.S., and Solomyak, M.Z. Quantitative analysis in Sobolev imbedding theorems and applications to spectral theory. In: Proceed. of X Summer Mathematical School. Yu.A. Mitropol'skiy and A.F. Shestopal (Eds), 1974, 5-189 (Russian); English transl. in: AMS Translations, Series 2, 114. AMS, Providence, R.I. 1980.

[2] Birman, M.S., and Solomyak, M.Z. Spectral theory of self-adjoint operators in Hilbert space, 2nd ed., revised and extended. Lan', St.Petersburg, 2010 [in Russian]; English transl. of the 1st ed.: Mathematics and Its Applications. Soviet Series. 5, Kluwer, Dordrecht etc. 1987.

[3] Nazarov, A.I. Spectral asymptotics for a class of integro-differential equations arising in the theory of fractional Gaussian processes. Preprint (2019), 31 pp. 